\title{
The impact of proximal cell rewiring compared with distal cell rewiring with single-stent deployment and subsequent kissing balloon inflation in the inadequate jailing of a Kaname stent on the side branch ostium
}

\author{
Fumiaki Nakao
}

Department of Cardiology, Yamaguchi Grand Medical Center, Yamaguchi, Japan

\begin{abstract}
Background: The aim of the study was to identify differences between proximal and distal cell rewiring with subsequent kissing balloon inflation (KBI) in the presence of a link connected to a carina on the jailed side branch ostium (SBO).

Methods: Kaname stents were deployed in bifurcation models $(n=12)$ with subsequent KBI and were confirmed by optical coherence tomography. The jailing configuration and cell rewiring were completely controlled and classified as follows: FC-Dist, free carina (no links connected to a carina) and distal cell rewiring; CC-Prox, connected to a carina (the presence of a link connected to a central carina) and proximal cell rewiring; CC-Dist, connected to a carina and distal cell rewiring; and FarDist, far-distal cell rewiring. Results: The number of frames with malapposed struts $(M S)$ in the SBO was significantly smaller in the CC-Prox group than in the CC-Dist group $(26.7 \pm 1.5$ and $39.7 \pm 0.6$, respectively, $p<0.05)$. The number of frames with MS continuously present from the distal end to the proximal side of the SBO was significantly smaller in the CC-Prox group than in the CC-Dist group $(17.0 \pm 2.0$ and $36.7 \pm$ \pm 5.8 , respectively, $p<0.05)$. The ratio of the maximal opened stent cell area to the SBO area was significantly larger in the CC-Prox group than in the CC-Dist group $(64.2 \pm 1.2 \%$ and $38.8 \pm 5.6 \%$, respectively, $p<0.05$ ).

Conclusions: This study showed that in cases with a link connected to a carina, fewer malapposed struts may be present in proximal cell rewiring with subsequent KBI than in distal cell rewiring. (Cardiol J 2018; 25, 3: 317-326)

Key words: coronary bifurcation, 3D-OCT, stent jail, recrossed cell, malapposed strut
\end{abstract}

\section{Introduction}

Coronary bifurcation lesions that include a left main bifurcation account for 15-20\% of all percutaneous coronary interventions (PCI) [1-3]. The development of new-generation drug-eluting stents and novel procedures has motivated interventional cardiologists to perform bifurcation PCI $[4,5]$. However, bifurcation PCI have a high risk of restenosis [6, 7], and adverse events that occur in patients who have undergone the left main bifurcation PCI are fatal [8].

The Ultimaster stent (Terumo, Tokyo, Japan), a 2-link, 8-crown, abluminal, bioabsorbable, polymer-coated, sirolimus-eluting, cobalt-chromium stent, with good trackability and interstrut-expandability, is one of the stents that is suitable for bifurcation lesions [9]. Its links are alternately

Address for correspondence: Dr. Fumiaki Nakao, Department of Cardiology, Yamaguchi Grand Medical Center, 10077 Ohsaki, Hofu, Yamaguchi 747-8511, Japan, tel: +81-835-22-4411, fax: +81-835-38-2210, e-mail: nakao-ymghp@umin.ac.jp 


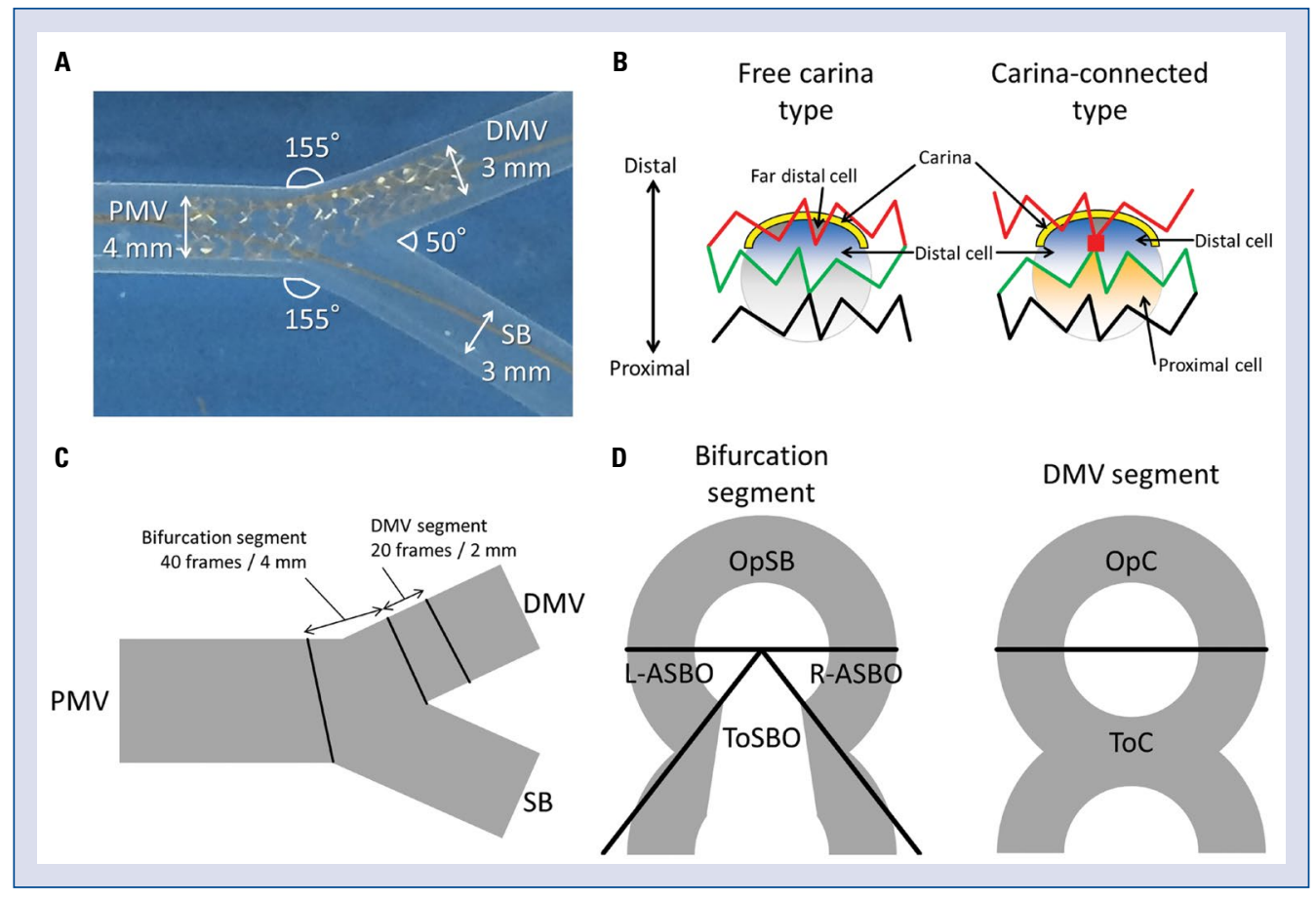

Figure 1. A. Bifurcation model; B. Classification of the configuration of overhanging struts in front of the side branch ostium (SBO) and definitions of the proximal and distal cells. The yellow band indicates the carina. The red square indicates the link at the center in the front of the SBO that connects to the stent strut (red line) landing the carina. The distal cell is defined as the area (blue area) enclosed by the carina and the stent strut (green line) that has at least one distal top of the stent hoop located in front of the SBO. The proximal cell is defined as the cell (orange area) present at one proximal side of the distal cell in front of the SBO. Far-distal cell rewiring is defined as rewiring to the area (arrows) enclosed by the carina and the stent strut (red line) that has no distal tops of the stent hoop located in front of the SBO. Longitudinal segmentation (C) and subsegmentation in the bifurcation segment and the distal main vessel (DMV) segment (D); PMV — proximal main vessel; SB — side branch; ToSBO — toward the SBO; OpSB — opposite the SB; L-ASBO — left-sided adjacent to the SBO; R-ASBO — right-sided adjacent to the SBO; ToC — toward the carina; OpC - opposite the carina.

located at $90^{\circ}$, which makes it easier to estimate the jailing configuration by checking the lateral view in a phantom study. The Kaname stent (Terumo) is a platform of the Ultimaster stent.

Frequency-domain optical coherence tomography (FD-OCT) is an imaging modality with high resolution and a high-speed pullback [10]. The relationships among stent struts, a side branch (SB) ostium (SBO), and a rewired cell can be observed in detail intraprocedurally via three-dimensional optical coherence tomography (3D-OCT), and the jailing configuration is classified as a free carina type (in which no stent links connect to a carina) or as a carina-connected type (in which stent links connect to a carina) [11]. The achievements of both the free carina type and distal rewiring have been reported to be associated with a lower incidence of malapposed struts (MS) [11]. However, in the presence of the carina-connected type, it is unclear which cell is better for rewiring with subsequent kissing balloon inflation (KBI). To identify differences between proximal and distal cell rewiring with subsequent KBI in terms of the carina-connected type, the jailing pattern and the rewired cell of a bifurcation model after the controlled deployment of a Kaname stent, and MS after KBI were investigated using FD-OCT and 3D-OCT.

\section{Methods}

\section{Bifurcation phantom vessel}

Uniform bifurcation phantom vessels made of polyvinyl chloride (Shounankasei, Hatano, Japan) were used, with a proximal main vessel (PMV) diameter of $4.0 \mathrm{~mm}$; a distal main vessel (DMV) diameter of $3.0 \mathrm{~mm}$; an SB diameter of $3.0 \mathrm{~mm}$; a main vessel angle of $155^{\circ}$; an SB angle of $155^{\circ}$; and a bifurcation angle of $50^{\circ}$ (Fig. 1A). 


\section{Procedures}

A phantom vessel was submerged in saline, and all of the procedures were performed under visual guidance. The first guidewire was inserted into a DMV through the PMV. A $3.0 \times 18 \mathrm{~mm}$ Kaname stent was deployed along the PMV and DMV matching the center of the stent to the SBO with a balloon inflation pressure of $4 \mathrm{~atm}$. The jailing configuration was controlled by gently pulling, pushing, or rotating the stent delivery system, and deployment pressure was raised to $9 \mathrm{~atm}$. The stent delivery balloon was then inflated three times, each time for $20 \mathrm{~s}$, to a balloon inflation pressure of $14 \mathrm{~atm}$. The proximal optimization technique was then performed with a $4.0 \times 8 \mathrm{~mm}$ non-compliant balloon. The second guidewire was recrossed through a target stent cell in front of the SBO under visual guidance, and SB dilation was performed with a $3.0 \times 15 \mathrm{~mm}$ semi-compliant balloon 3 times, each time for $20 \mathrm{~s}$, balloon inflation pressure was $6 \mathrm{~atm}$ according to the compliance chart. KBI was then performed 3 times [12] for 20 s each using two $3.0 \times 15 \mathrm{~mm}$ semi-compliant balloons with minimal overlapping [13] and balloon inflation pressures of $6 \mathrm{~atm}$. The balloon diameters for KBI were determined by Finet's formula: $0.678 \times(3.0 \mathrm{~mm}+3.0 \mathrm{~mm})=4.02 \mathrm{~mm}$ [14]

According to a report by Okamura et al. [11], the jailing patterns of the SBO were estimated in a lateral visual view, confirmed by 3D-OCT (as mentioned below), and were divided into two types as follows (Fig. 1B): a free carina type, in which the stent strut (red line) that landed the carina had no link in front of the SBO, and a carina-connected type, in which the stent strut (red line) that landed the carina and had a link (red square) at the center in the front of the SBO [11, 15]. Distal cell rewiring was defined as rewiring to the area (blue area) that was enclosed by the carina and stent strut (green line) that had at least one distal top of the stent hoop located in front of the SBO (Fig. 1B) $[11,15]$. Proximal cell rewiring was defined as rewiring to the cell (orange area) that was present at one proximal side of the distal cell in front of the SBO (Fig. 1B) $[11,15]$. Far-distal cell rewiring was defined as rewiring to the area (Fig. 1B, arrow) that was enclosed by the carina and the stent strut (red line) that had no distal tops of the stent hoop located in front of the SBO [16].

Twelve phantom vessels were studied and divided into four groups: FC-Dist, free carina type and distal cell rewiring $(\mathrm{n}=3)$; CC-Prox, carinaconnected type and proximal cell rewiring $(n=3)$;
CC-Dist, carina-connected type and distal cell rewiring $(\mathrm{n}=3)$; and FarDist, far-distal cell rewiring $(\mathrm{n}=3)$.

\section{FD-OCT}

FD-OCT data were obtained at a pullback speed of $18 \mathrm{~mm} / \mathrm{s}(0.1 \mathrm{~mm} /$ frame $)$ with the Dragonfly JP intravascular imaging catheter (St. Jude Medical, Minneapolis, MN, USA) and the ILUMIEN Optis FD-OCT imaging system (St. Jude Medical). FD-OCT images were analyzed with proprietary software (St. Jude Medical) by an experienced analyst. Struts were classified as MS if the distance between the strut maker and the lumen contour was larger than the specific strut thickness of the Kaname $(80 \mu \mathrm{m})$ plus conventional axial resolution of OCT $(14 \mu \mathrm{m})[11,17]$ in cross-sectional images from an FD-OCT pullback in the stented vessel. Non-apposed struts over the SBO (NASB) should be differentiated from MS because the degree of neointimal coverage over NASB and MS which may slightly differ [17]. However, both uncovered MS and uncovered NASB can result in adverse events. In this study, NASB was included in MS.

The numbers of apposed struts and MS after KBI were counted at every cross-section (40 frames) in the bifurcation segment (divided into 4 subsegments: ToSBO, toward the SBO; OpSB, opposite the SB; L-ASBO, left-sided adjacent to the $\mathrm{SBO}$; R-ASBO, right-sided adjacent to the SBO), and in the distal segment (divided into two $180^{\circ}$ halves toward or opposite the carina: $\mathrm{ToC}, \mathrm{OpC}$ ) that extended from the carina tip to 20 frames of a distal side (Fig. 1C, D) [18]. The number of frames with MS was counted in each subsegment. The metal carina index was defined as the number of frames with MS continuously present from the distal end of the SBO to the proximal side.

\section{D-OCT}

The jailing pattern and a rewired cell were confirmed intraprocedurally via $3 \mathrm{D}-\mathrm{OCT}$. FD-OCT image data were transferred to an off-line workstation with USB flush drive. Vaa3D v2.8 (3D reconstruction freeware, Howard Hughes Medical Institute, Chevy Chase, MD, USA) was used to visualize 3D-OCT [19]. The jailing pattern and rewired cell were confirmed with a longitudinal cutaway view of 3D-OCT and were classified as above (Fig. 1B). The jailing strut opening was measured to provide the ratio of maximal opened cell area to the SBO area with a 3D-OCT image that displayed the SBO from the SB side. 
Table 1. Number of frames with malapposed struts.

\begin{tabular}{lcccc}
\hline Subsegment & $\begin{array}{c}\text { FC-Dist } \\
(\mathbf{n}=3)\end{array}$ & $\begin{array}{c}\text { CC-Prox } \\
(\mathbf{n}=3)\end{array}$ & $\begin{array}{c}\text { CC-Dist } \\
(\mathbf{n}=3)\end{array}$ & $\begin{array}{c}\text { FarDist } \\
(\mathbf{n}=1)\end{array}$ \\
\hline ToC (n/20 frames) & $1.0 \pm 1.7$ & $0.7 \pm 1.2$ & $3.3 \pm 2.9$ & 14 \\
OpC (n/20 frames) & $0.0 \pm 0.0$ & $1.3 \pm 2.3$ & $1.3 \pm 2.3$ & 6 \\
ToSBO (n/40 frames) & $22.7 \pm 3.5$ & $26.7 \pm 1.5$ & $39.7 \pm 0.6^{* \#}$ & 38 \\
L-ASBO (n/40 frames) & $3.0 \pm 5.2$ & $4.3 \pm 4.0$ & $5.0 \pm 1.0$ & 0 \\
R-ASBO (n/40 frames) & $12.0 \pm 12.5$ & $12.7 \pm 6.4$ & $10.0 \pm 12.2$ & 13 \\
OpSB (n/40 frames) & $8.3 \pm 11.0$ & $6.3 \pm 6.7$ & $19.7 \pm 7.2^{\#}$ & 21 \\
\hline
\end{tabular}

Data are expressed as the means \pm standard deviations; ${ }^{*} p<0.05$ vs. FC-Dist, ${ }^{*} p<0.05$ vs. CC-Prox; FC-Dist - free carina type and distal cell rewiring; CC-Prox - carina-connected type and proximal cell rewiring; CC-Dist — carina-connected type and distal cell rewiring; FarDist - far-distal cell rewiring; ToC — toward the carina; OpC — opposite the carina; ToSBO — toward the side branch (SB) ostium (SBO); L-ASBO — left-sided adjacent to the SBO; R-ASBO — right-sided adjacent to the SBO; OpSB — opposite the SB

Table 2. The metal carina index, number of malapposed struts, and jailing strut opening.

\begin{tabular}{lcccc}
\hline & $\begin{array}{c}\text { FC-Dist } \\
(\mathbf{n}=3)\end{array}$ & $\begin{array}{c}\text { CC-Prox } \\
(\mathbf{n}=3)\end{array}$ & $\begin{array}{c}\text { CC-Dist } \\
(\mathbf{n}=3)\end{array}$ & $\begin{array}{c}\text { FarDist } \\
(\mathbf{n}=1)\end{array}$ \\
\hline Metal carina index & $4.7 \pm 4.5$ & $17.0 \pm 2.0^{*}$ & $36.7 \pm 5.8^{* \#}$ & 20 \\
Number of malapposed struts in the ToSBO (n) & $55.3 \pm 2.3$ & $66.3 \pm 7.0^{*}$ & $93.3 \pm 12.4^{* *}$ & 91 \\
Jailing struts opening (\%) & $72.2 \pm 4.3$ & $64.2 \pm 1.2^{*}$ & $38.8 \pm 5.6^{* \#}$ & 35 \\
\hline
\end{tabular}

Data are expressed as the means \pm standard deviations; ${ }^{*} p<0.05$ vs. FC-Dist, ${ }^{*} p<0.05$ vs. CC-Prox; the metal carina index is defined as the number of frames with malapposed struts continuously present from the distal end of the side branch ostium (SBO) to the proximal side. The jailing struts opening is defined as the ratio of maximal opened cell area to the side branch ostial area; FC-Dist - free carina type and distal cell rewiring; CC-Prox - carina-connected type and proximal cell rewiring; CC-Dist — carina-connected type and distal cell rewiring;

FarDist — far-distal cell rewiring; ToSBO — toward the SBO

\section{Statistical analysis}

Variables are expressed as means \pm standard deviation and were compared using the Mann-Whitney U test. A p-value of less than 0.05 was considered significant. All data were calculated and analyzed using StatView software (Version 5.0J for Windows, SAS Institute, Cary, NC, USA).

\section{Results}

Controls of both the jailing type and rewired cell were successful in all cases. Procedures were completed in FC-Dist, CC-Prox, and CC-Dist groups. Two cases in the FarDist group were not completed because the struts on the DMV segment were pulled to the SB segment by SB dilation. Therefore, it was not possible to include the FarDist group in the statistical analysis.

Table 1 shows the numbers of frames with MS in FC-Dist, CC-Prox, and CC-Dist groups. The numbers of frames with MS in ToC, OpC, L-ASBO, and R-ASBO segments were not significantly different in FC-Dist, CC-Prox, and CC-Dist groups. The number of frames with MS in the ToSBO segment was significantly larger in the CC-Dist group than in FC-Dist and CC-Prox groups (FC-Dist, $22.7 \pm$ \pm 3.5 ; CC-Prox, $26.7 \pm 1.5$; CC-Dist, $39.7 \pm 0.6^{* \#}$;
${ }^{*} \mathrm{p}<0.05$ vs. FC-Dist, ${ }^{*} \mathrm{p}<0.05$ vs. CC-Prox). The number of frames with MS in OpSB segment was significantly larger in CC-Dist group than in CC-Prox group (FC-Dist, $8.3 \pm 11.0$; CC-Prox, $6.3 \pm$ \pm 6.7 ; CC-Dist, $19.7 \pm 7.2^{\#}$; ${ }^{\#} \mathrm{p}<0.05$ vs. CC-Prox). The number of frames with MS in the bifurcation segment and jailing strut opening in a FarDist case were similar to those in the CC-Dist group, but the number of frames with MS in the DMV segment was larger than in other groups.

Table 2 shows the metal carina index, the number of MS in the ToSBO segment, and the jailing strut opening in the FC-Dist, CC-Prox, and CC-Dist groups. The metal carina index was significantly larger in the CC-Prox group compared with the FC-Dist group and in the CC-Dist group compared with FC-Dist and CC-Prox groups (FC-Dist, $4.7 \pm 4.5$; CC-Prox, $17.0 \pm 2.0^{*}$; CC-Dist, $36.7 \pm 5.8^{* \#} ;{ }^{*} \mathrm{p}<0.05$ vs. FC-Dist, ${ }^{*} \mathrm{p}<0.05$ vs. CC-Prox). The number of MS in the ToSBO segment was significantly smaller in the FC-Dist group compared with the CC-Prox group and significantly larger in the CC-Dist group compared with FC-Dist and CC-Prox groups (FC-Dist, $55.3 \pm 2.3$; CC-Prox, $66.3 \pm 7.0^{*}$; CC-Dist, $93.3 \pm 12.4^{* *}$; *p $<0.05$ vs. FC-Dist, ${ }^{\#} \mathrm{p}<0.05$ vs. CC-Prox). The jailing strut opening was significantly larger in 


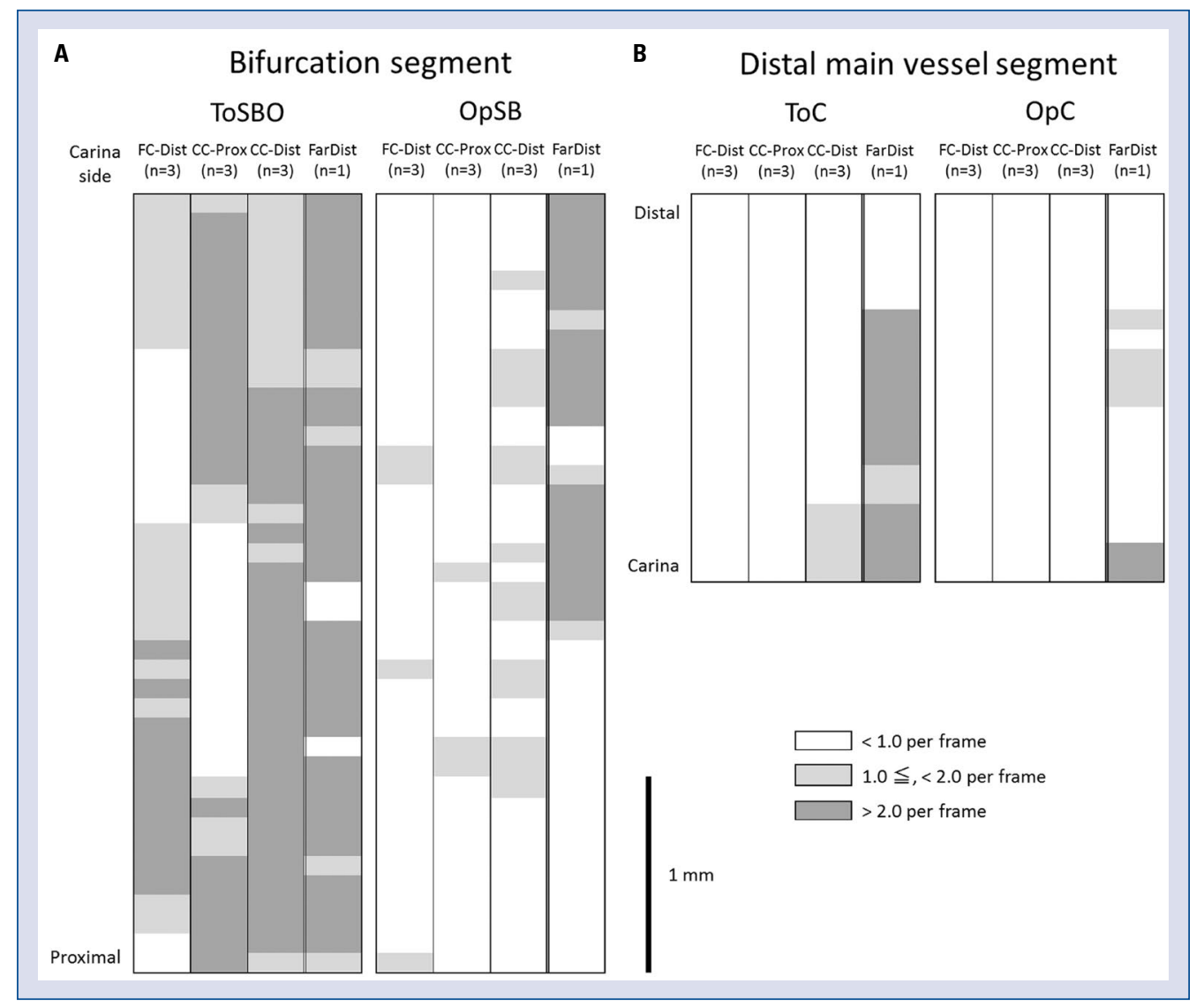

Figure 2. Distribution map of the mean number of malapposed struts per frame; A. Bifurcation segment; B. Distal main vessel segment; ToSBO — toward the side branch (SB) ostium; OpSB — opposite the SB; ToC — toward the carina; $\mathrm{OpC}$ - opposite the carina; FC-Dist — free carina type and distal cell rewiring; CC-Prox — carina-connected type and proximal cell rewiring; CC-Dist — carina-connected type and distal cell rewiring; FarDist — far-distal cell rewiring.

the FC-Dist group compared with CC-Prox and CC-Dist groups and in the CC-Prox group compared with the CC-Dist group (FC-Dist, $72.2 \pm 4.3 \%$; CC-Prox, $64.2 \pm 1.2 \%$; ;C-Dist, $38.8 \pm 5.6 \%{ }^{* \#}$; ${ }^{*} \mathrm{p}<0.05$ vs. FC-Dist, ${ }^{*} \mathrm{p}<0.05$ vs. CC-Prox).

Figure 2 shows the distribution map of the mean number of MS per frame. In the ToSBO segment, though few MS were found near the carina in the FC-Dist group and around the center of the SBO in the CC-Prox group, MS were present all over the $\mathrm{SBO}$ in the CC-Dist group and the FarDist group. The MS were larger in the OpSB segment in CC-Dist and FarDist groups compared with those in the FC-Dist and CC-Prox groups. In the FarDist group, many MS were detected in the ToC segment.

Representative cases of each group are shown in Figure 3 (FC-Dist), Figure 4 (CC-Prox), Figure 5 (CC-Dist), and Figure 6 (FarDist). The typical metal carina is shown in CC-Prox (Fig. 4G). 3D-OCT indicated that deformed residual struts are present from the proximal end to the distal end on the SBO in CC-Dist and FarDist groups (Fig. 5G, 6G). The struts on the DMV segment are pulled to the SB segment in a failed case in FarDist (Fig. 6H, 6I).

\section{Discussion}

The present study demonstrated that proximal cell rewiring with subsequent $\mathrm{KBI}$ in the presence of a link that connected to a carina with a Kaname stent was better than distal cell rewiring with respect to MS, the metal carina, and the inter-strut opening in the SBO.

Single-stent deployment is generally recommended for a bifurcation lesion [6]. The results of previous clinical studies of single-stent deployment and subsequent KBI might not show the same trend because in those studies, these objects were not classified in detail (sites of the branch, bifurcation angles, vessel diameters, types of stents, the jailing type, a rewired cell, methods of KBI, etc.) 


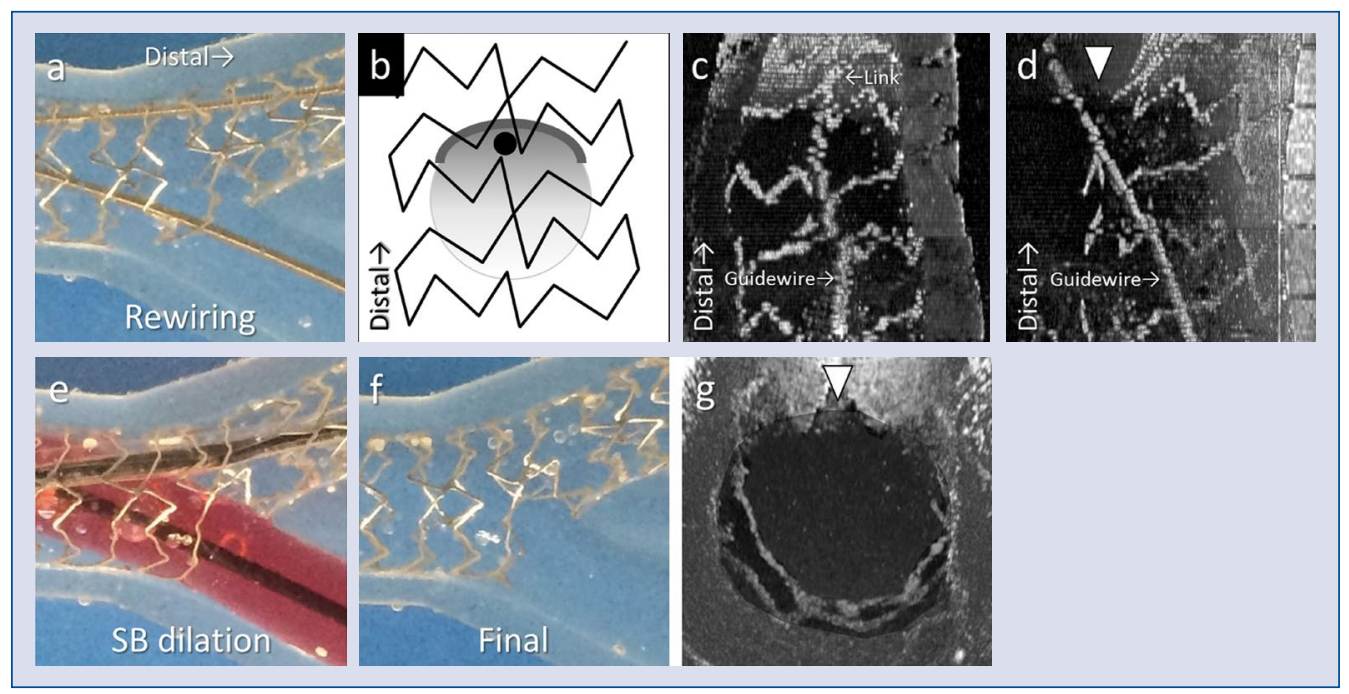

Figure 3. Representative case of the free carina type and distal cell rewiring (FC-Dist). A. Photograph showing rewiring to the side branch (SB) after the proximal optimization technique; B. Schematic showing rewiring facing the SB ostium (SBO) (closed circle indicates rewired cell). Three-dimensional optical coherence tomography (3D-OCT) facing the SBO (C) and perpendicular to the SBO (D) with a longitudinal cut-away view. (E) SB dilation; F. Final photograph after kissing balloon inflation; G. Final SBO viewed from the SB side by 3D-OCT; arrowhead — carina.

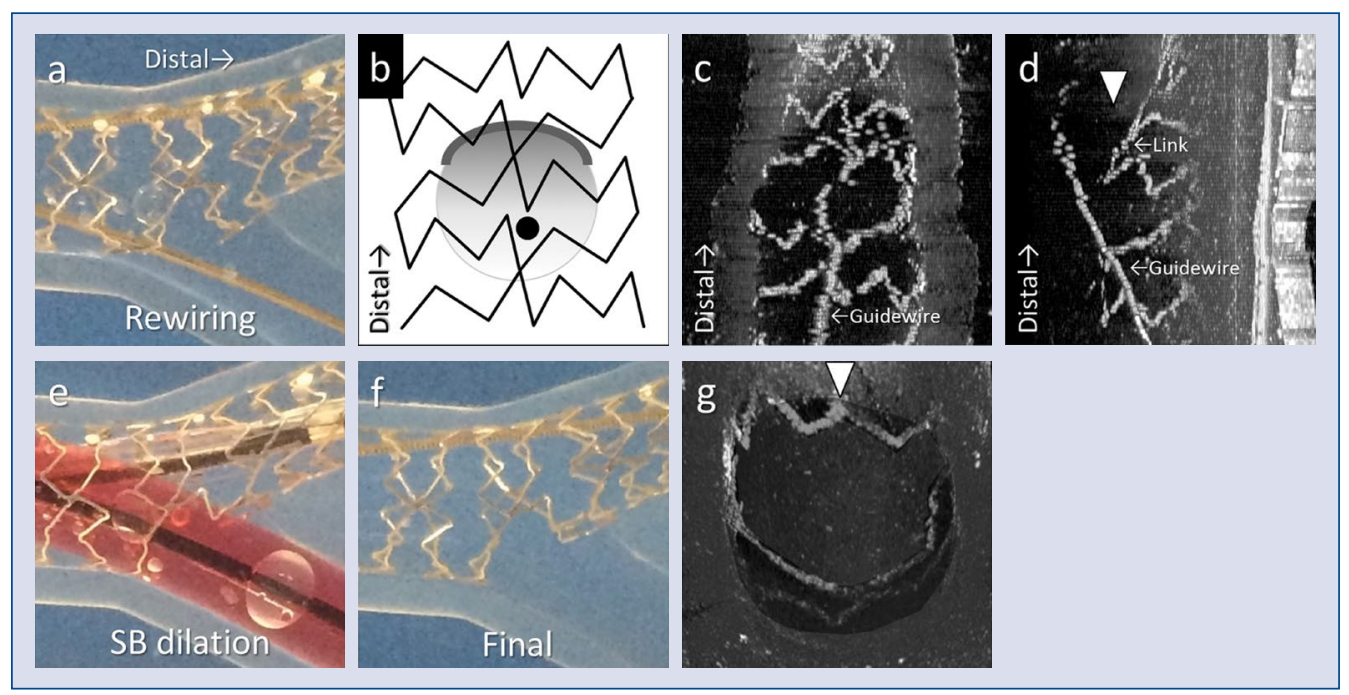

Figure 4. A-G. Representative case of the carina-connected type and proximal cell rewiring (CC-Prox). Descriptions and abbreviations are the same as in Figure 3.

$[5,7,20]$. In a previous study, the jailing type was not checked and the distal side rewiring was performed. A previous bench test that used a bifurcation model showed that a two-link stent was more deformed than a three-link stent [13]. Two-link stents have more trackability and more interstrutexpandability than three-link stents, but they are weaker longitudinally. In a previous report that described the superiority of distal cell rewiring, closed-cell or three-link stents were often used, but jailing configurations were not considered [21]. An appropriately rewired cell of a two-link stent may differ from those of closed-cell or three-link stents, whereas an appropriately rewired cell in the carina-connected type of two-link stent may not be a distal cell, as in this study. Jailing configurations can be checked intraprocedurally in a $3 \mathrm{D}-\mathrm{OCT}$ era, and therefore, the selection of a rewired cell, 


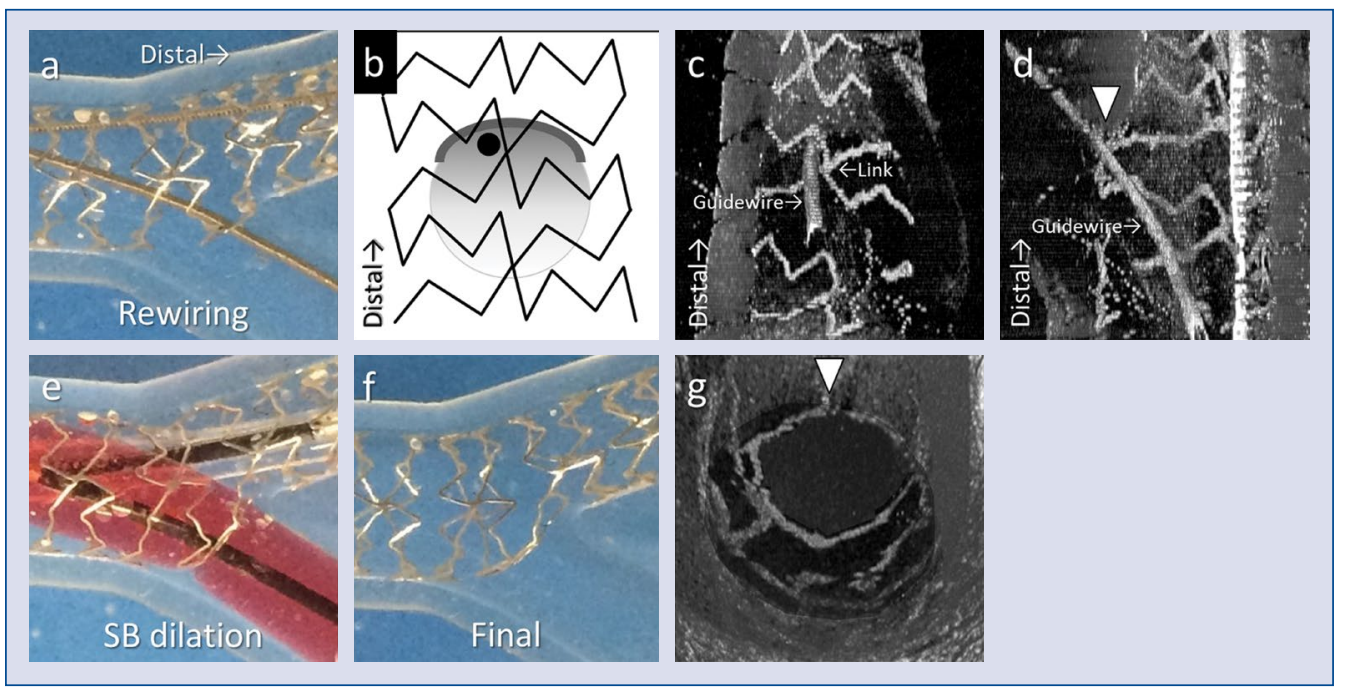

Figure 5. A-G. Representative case of the carina-connected type and distal cell rewiring (CC-Dist). Descriptions and abbreviations are the same as in Figure 3.

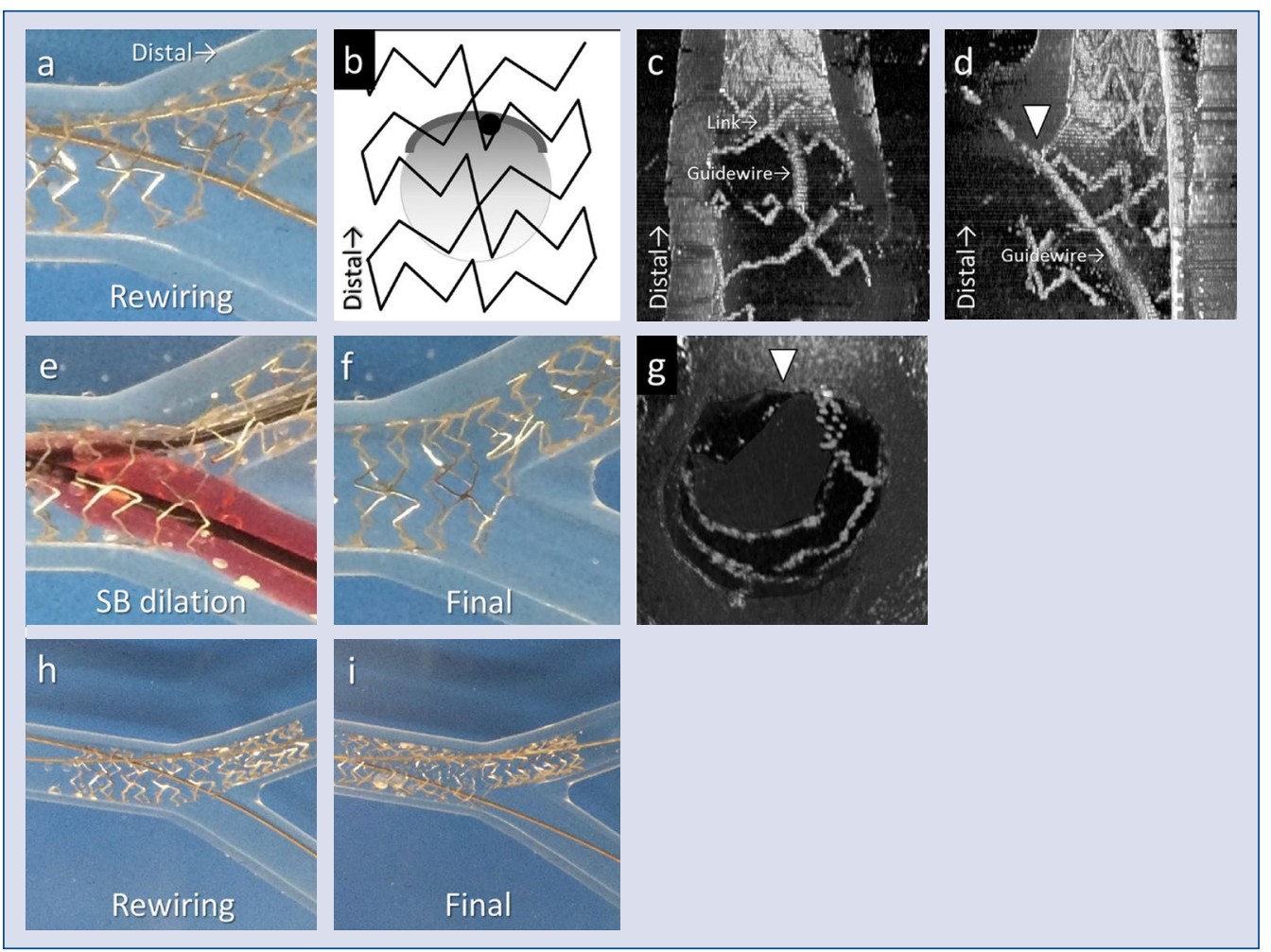

Figure 6. Representative case of far-distal cell rewiring (FarDist). Descriptions of $\mathbf{A}-\mathbf{G}$ and abbreviations are the same as in Figure 3. Photograph showing rewiring to the side branch after the proximal optimization technique $(\mathbf{H})$ and final photograph after kissing balloon inflation (I) in a failed case of FarDist.

depending on the type of the jailing configuration, may be required.

A gentle bifurcation angle, such as the parallel type in the classification that used 3D-OCT, leads to a compromised PCI [22] and has problems related to carina shift and maximum stent dilatation diameter. Therefore, to simulate poorer conditions, a uniform bifurcation model with a gentle bifurca- 
tion angle was used in the present study, which may have led to a comparatively large number of MS in the SBO even in the FC-Dist group. According to a clinical study that used a two-link biolimus-eluting stent, a steeper angle of the main (to-be-stented) vessel results in a less frequent presence of carina-connected type [15]; nonetheless, the carinaconnected type is present in approximately $46 \%$ of general clinical cases $[11,15]$. In this study, with complete control of the jailing configuration, MS and deformed struts were present in the bifurcation segment in CC-Dist and FarDist groups as well as in the DMV segment. The CC-Prox group had a larger metal carina than the FC-Dist group, but other factors in the CC-Prox group were mostly favorable. A metal carina leads to high shear stress, but may also lead to better results than MS all over the SBO, as observed in the CC-Dist group. Approximately half of clinical cases may require proximal cell rewiring but not distal side rewiring.

Two of three cases in the FarDist group were not completed because the struts on the DMV segment were pulled to the SB segment by SB dilation, which indicated that far-distal cell rewiring leads to compromised results. No additional studies for an incomplete procedure in the FarDist group were planned to compensate for this outcome, and therefore, a statistical analysis that included the FarDist group could not be performed. In the FarDist group $(\mathrm{n}=1)$, MS occurred in almost all of the segments except L-ASBO and R-ASBO, the metal carina index was large, and jailing strut opening was small. Notably, the number of frames with MS was extremely large in the DMV segment. With other two-link stents, the presence of the carina-connected type and either distal cell or far-distal cell rewiring can also lead to NASB and longitudinal deformed struts on a DMV [16]. These compromised sites may be the same as the common restenosis sites in single-stent deployment and subsequent KBI [20].

Some cases require two-stent deployments [6]. For instance, NASB and SB dilation after the first stent deployment may influence MS after the completion of culotte stenting, whereas far-distal cell rewiring might increase the risk of abluminal rewiring of the SB stent [23]. Through the use of 3D-OCT, it was reported that NASB might be diminished if the jailing configuration and the rewiring position were proper in culotte stenting [11]. Moreover, in a case with NASB after the first stent deployment, strategies can be changed or a second stent deployment can be avoided with the use of 3D-OCT guidance [24].
The use of re-proximal optimization technique (Re-POT) without KBI has recently been proposed [25]. Re-POT, which was not performed in the present study, might reduce MS in proximal ToSBO. Although the advantages and disadvantages of KBI were not considered in this study, the struts in $\mathrm{OpSB}$ and $\mathrm{OpC}$ in the CC-Dist and FarDist groups were deformed and malapposed by SB dilation (Fig. 5E, 6E); therefore, a balloon for a main vessel may not be crossed in the same clinical situation. At the very least, the insertion of a balloon for rePOT prior to SB dilation may be needed.

According to the present study, when SB dilation is needed in the presence of carina-connected type with a Kaname/Ultimaster stent, proximal cell rewiring may be recommended over distal cell rewiring because of fewer MS and fewer deformed struts even in cases involving a metal carina. Similarly to a previous study [11], the present study indicated that FC-Dist group was better than other situations. To constantly obtain a free carina type, development of a specific stent structure that does not lead to a carina-connected link and novel techniques of stent deployment are desired.

\section{Limitations of the study}

Parts of the guidewire shadow cannot be observed in FD-OCT studies. It has been reported that a GW with a sparse spring coil (e.g. JOKER, Japan Lifeline, Tokyo, Japan) is useful for the reduction of the guidewire shadow on FD-OCT [26]. Clinical cases include various vessel diameters and bifurcation angles; the present study was, however, an evaluation using a uniform phantom vessel with no stenoses. A phantom vessel wall differs from a real vessel wall in terms of hardness and friction. The Kaname stent does not have an abluminal polymer, and it is unclear whether the behavior of the Ultimaster stent is the same as that of the Kaname stent. Because MS in a strictly controlled jailing type and a rewired cell were considered using a single type of a stent and a phantom vessel, these results might be meaningful even if the number of cases in each group was small. In the carina-connected type, the position of the link was restricted to the center, and the lateral subtype was not considered. From the perspective of jailing type and rewired cell, larger studies involving clinical cases that have been treated with various types of stents are needed to validate the present results.

\section{Conclusions}

This study indicated that, in the presence of a carina-connected link in front of a jailed SBO with 
a Kaname stent, MS for proximal cell rewiring with subsequent KBI may be smaller than MS for distal cell rewiring with subsequent KBI, and fardistal cell rewiring should be strictly avoided. In clinical cases, it is important to confirm the jailing configuration and the rewired cell intraprocedurally via $3 \mathrm{D}-\mathrm{OCT}$. These insights may help guide adequate rewiring in inadequate jailing with the Kaname stent.

\section{Acknowledgements}

The author would like to thank Tooru Ueda, Takamasa Oda, Masashi Kanemoto, Yasuhiro Ikeda, and Takashi Fujii of the Department of Cardiology of Yamaguchi Grand Medical Center for their support. The author would also like to thank Jutaro Yamada, Takayuki Okamura, and Masafumi Yano of the Division of Cardiology, Department of Medicine and Clinical Science of Yamaguchi University Graduate School of Medicine for their helpful advice.

Conflict of interest: F. Nakao received honoraria for technical consulting from Terumo. This article does not contain any studies with human participants or animals.

\section{References}

1. Iakovou I, Ge L, Colombo A. Contemporary stent treatment of coronary bifurcations. J Am Coll Cardiol. 2005; 46(8): 1446-1455, doi: 10.1016/j.jacc.2005.05.080, indexed in Pubmed: 16226167.

2. Ferrante G, Presbitero P, Valgimigli M, et al. Percutaneous coronary intervention versus bypass surgery for left main coronary artery disease: a meta-analysis of randomised trials. EuroIntervention. 2011; 7(6): 738-746, 1, indexed in Pubmed: 21947626.

3. De Caterina AR, Cuculi F, Banning AP. Incidence, predictors and management of left main coronary artery stent restenosis: a comprehensive review in the era of drug-eluting stents. EuroIntervention. 2013; 8(11): 1326-1334, doi: 10.4244/EIJV8I11A201, indexed in Pubmed: 23538159.

4. Hildick-Smith D, Lassen J, Koo BK. One or two stents for coronary bifurcation lesions? EuroIntervention. 2010; 6(J): J61-J64, doi: 10.4244/eijv6supja10.

5. Biondi-Zoccai G, Sheiban I, De Servi S, et al. To kiss or not to kiss? Impact of final kissing-balloon inflation on early and longterm results of percutaneous coronary intervention for bifurcation lesions. Heart Vessels. 2014; 29(6): 732-742, doi: 10.1007/ s00380-013-0416-0, indexed in Pubmed: 24077644.

6. Yamashita T, Nishida T, Adamian MG, et al. Bifurcation lesions: two stents versus one stent--immediate and follow-up results. J Am Coll Cardiol. 2000; 35(5): 1145-1151, indexed in Pubmed: 10758954.

7. Song YB, Hahn JY, Choi SH, et al. Sirolimus- versus paclitaxeleluting stents for the treatment of coronary bifurcations results: from the COBIS (Coronary Bifurcation Stenting) Registry. J Am Coll Cardiol. 2010; 55(16): 1743-1750, doi: 10.1016/j. jacc.2010.02.008, indexed in Pubmed: 20394879.
8. Park SM, Ahn CM, Hong SJ, et al. Acute changes of left ventricular hemodynamics and function during percutaneous coronary intervention in patients with unprotected left main coronary artery disease. Heart Vessels. 2015; 30(4): 432-440, doi: 10.1007/ s00380-014-0495-6, indexed in Pubmed: 24633497.

9. Orvin K, Carrie D, Richardt G, et al. Comparison of sirolimus eluting stent with bioresorbable polymer to everolimus eluting stent with permanent polymer in bifurcation lesions: Results from CENTURY II trial. Catheter Cardiovasc Interv. 2016; 87(6): 1092-1100, doi: 10.1002/ccd.26150, indexed in Pubmed: 26268482 .

10. Okamura T, Onuma Y, Garcia-Garcia HM, et al. High-speed intracoronary optical frequency domain imaging: implications for three-dimensional reconstruction and quantitative analysis. EuroIntervention. 2012; 7(10): 1216-1226, doi: 10.4244/EIJV7I10A194, indexed in Pubmed: 22334321.

11. Okamura T, Onuma Y, Yamada J, et al. 3D optical coherence tomography: new insights into the process of optimal rewiring of side branches during bifurcational stenting. EuroIntervention. 2014; 10(8): 907-915, doi: 10.4244/EIJV10I8A157, indexed in Pubmed: 24531393.

12. Hikichi Y, Umezu M, Node K, et al. Reduction in incomplete stent apposition area caused by jailed struts after single stenting at left main bifurcation lesions: micro-CT analysis using a threedimensional elastic bifurcated coronary artery model. Cardiovasc Interv Ther. 2017; 32(1): 12-17, doi: 10.1007/s12928-016-0380-6, indexed in Pubmed: 26968169.

13. Murasato Y, Iwasaki K, Yamamoto T, et al. Optimal kissing balloon inflation after single-stent deployment in a coronary bifurcation model. EuroIntervention. 2014; 10(8): 934-941, doi: 10.4244/EIJV10I8A160, indexed in Pubmed: 24531354.

14. Finet G, Gilard M, Perrenot B, et al. Fractal geometry of arterial coronary bifurcations: a quantitative coronary angiography and intravascular ultrasound analysis. EuroIntervention. 2008; 3(4): 490-498, indexed in Pubmed: 19736093.

15. Nakao F, Okamura T, Suetomi T, et al. Differences of side branch jailing between left main-left anterior descending artery stenting and left main-left circumflex artery stenting with Nobori biolimus-eluting stent. Heart Vessels. 2016; 31(12): 1895-1903, doi: 10.1007/s00380-016-0812-3, indexed in Pubmed: 26879743.

16. Nakao F. Importance of confirmation by instant stent-accentuated three-dimensional optical coherence tomography during bifurcation stenting: far distal rewiring of iSA3D-OCT. AsiaIntervention. 2015; $1: 71$.

17. Gutiérrez-Chico JL, Wykrzykowska J, Nüesch E, et al. Delayed coverage in malapposed and side-branch struts with respect to well-apposed struts in drug-eluting stents: in vivo assessment with optical coherence tomography. Circulation. 2011; 124(5): 612-623, doi: 10.1161/CIRCULATIONAHA.110.014514, indexed in Pubmed: 21768536.

18. Hariki H, Shinke T, Otake H, et al. Potential benefit of final kissing balloon inflation after single stenting for the treatment of bifurcation lesions--insights from optical coherence tomography observations. Circ J. 2013; 77(5): 1193-1201, indexed in Pubmed: 23446003.

19. Nakao F, Ueda T, Nishimura S, et al. Novel and quick coronary image analysis by instant stent-accentuated three-dimensional optical coherence tomography system in catheterization laboratory. Cardiovasc Interv Ther. 2013; 28(3): 235-241, doi: 10.1007/ s12928-013-0161-4, indexed in Pubmed: 23355032. 
20. Niemelä M, Kervinen K, Erglis A, et al. Nordic-Baltic PCI Study Group. Randomized comparison of final kissing balloon dilatation versus no final kissing balloon dilatation in patients with coronary bifurcation lesions treated with main vessel stenting: the Nordic-Baltic Bifurcation Study III. Circulation. 2011; 123(1): 79-86, doi: 10.1161/CIRCULATIONAHA.110.966879, indexed in Pubmed: 21173348.

21. Alegría-Barrero E, Foin N, Chan PH, et al. Optical coherence tomography for guidance of distal cell recrossing in bifurcation stenting: choosing the right cell matters. EuroIntervention. 2012; 8(2): 205-213, doi: 10.4244/EIJV8I2A34, indexed in Pubmed: 22581489.

22. Farooq V, Serruys PW, Heo JHo, et al. New insights into the coronary artery bifurcation hypothesis-generating concepts utilizing 3-dimensional optical frequency domain imaging. JACC Cardiovasc Interv. 2011; 4(8): 921-931, doi: 10.1016/j.jcin.2011.06.004, indexed in Pubmed: 21851908.

23. Murasato Y, Hikichi Y, Horiuchi M. Examination of stent deformation and gap formation after complex stenting of left main coronary artery bifurcations using microfocus computed tomography. J Interv Cardiol. 2009; 22(2): 135-144, doi: 10.1111/j.15408183.2009.00436.x, indexed in Pubmed: 19379472.

24. Nakao F. Instant stent-accentuated 3-dimensional optical coherence tomography of struts pulled up by pulling stuck wire and folded by kissing balloon dilation. JACC Cardiovasc Interv. 2015; 8(8): e125-e126, doi: 10.1016/j.jcin.2015.01.038, indexed in Pubmed: 26117467.

25. Finet G, Derimay F, Motreff P, et al. Comparative analysis of sequential proximal optimizing technique versus kissing balloon inflation technique in provisional bifurcation stenting: fractal coronary bifurcation bench test. JACC Cardiovasc Interv. 2015; 8(10): 1308-1317, doi: 10.1016/j.jcin.2015.05.016, indexed in Pubmed: 26315733.

26. Nakao F, Ueda T, Nishimura S, et al. Guide wire shadow assessed by shading index is reduced in sparse spring coil wire in optical coherence tomography. Cardiovasc Interv Ther. 2013; 28(4): 362-367, doi: 10.1007/s12928-013-0186-8, indexed in Pubmed: 23670441. 